\title{
Local estimation of thermal effusivity by stimulated infrared thermography
}

By Kamel Mouhoubi*, Jean-Luc Bodnar*, Jean-Marc Vallet ${ }^{* *}$, Vincent Detalle***

*GRESPI / CATHERM, UFR Sciences Exactes et Naturelles, BP 1039, 51687 Reims cedex 02 (orcid.org/0000-0002-4880-8792)

${ }^{\star *}$ Centre Interdisciplinaire de Conservation et Restauration du Patrimoine (CICRP), 21 rue Guibal, 13003 Marseille

${ }^{* * *}$ C2RMF, Palais du Louvre - Porte des Lions, 14, quai François Mitterrand, 75001 Paris

\begin{abstract}
Our laboratory specializes in the study of alterations affecting works of art such as frescoes[1,2]. Thermal effusivity is a thermophysical quantity that allows us to detect alterations or to highlight contamination of chemical species in these works such as hygroscopic salts. The two approaches presented here, based on the analysis of lateral heat scattering following local laser excitation, make it possible to arrive at a good estimate of this thermophysical quantity, independently of the thickness of the samples and without taking samples (which is an advantage for the analysis of works of art).
\end{abstract}

Keywords : thermography, stimulated infrared, thermal characterization, effusivity, non-destructive testing, laser

\section{Nomenclature}

a Thermal diffusivity, $\mathrm{m}^{2} \cdot \mathrm{s}^{-1}$

$b \quad$ Thermal effusivity, J.K. $\mathrm{K}^{-1} \cdot \mathrm{m}^{-2} \cdot \mathrm{s}^{-1 / 2}$

c Thermal capacity, $\mathrm{J}^{\mathrm{kg}} \mathrm{kg}^{-1} \cdot \mathrm{K}^{-1}$

F Fourier Transform

H Hankel Transform

I(t) Surface integral, K

$Q \quad$ Total energy (of the beam), J

Q' Term equivalent to an energy density, J.m-1

$\mathrm{R} \quad$ Laser spot radius, $\mathrm{m}$

$r \quad$ Cylindrical coordinate, $m$

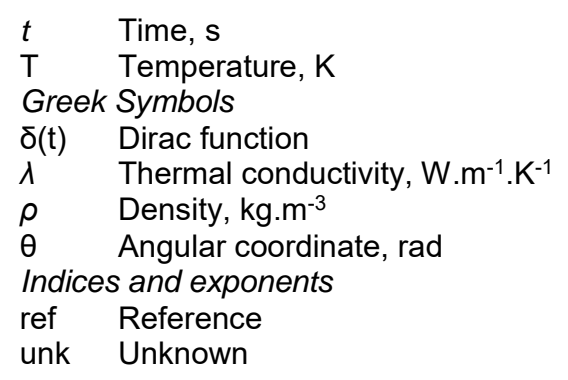




\section{Introduction}

Our laboratory has been working for more than 15 years in the field of the conservation of cultural heritage by stimulated infrared thermography. After having shown the possibilities of the method in terms of detection of structural defects, we are currently working on the detection of pollution by hygroscopic salts. The approach taken is to try to detect these pollutants by thermal analysis. The idea is that the presence of salts modifies the local thermophysical properties of works of art. In the case of wall paintings, the thickness of the samples does not allow the use of transverse methods for estimating thermophysical properties. We then turned to the use of a longitudinal measurement method. The latter involves local laser excitation and the study of lateral heat diffusion. In this work, we show that this approach allows a good approximation of the thermal effusivity parameter. Our presentation is divided into four stages: We present the theoretical approach of our study, we then present the samples studied and the experimental system implemented, and finally, we present the results obtained.

\section{The theoretical model developed for the study}

The method of operation of the longitudinal thermal effusivity estimation method developed for the study is as follows - see also [3] : A sample is subjected on its front face to localized laser excitation. This excitation is temporally close to a Dirac delta $\delta(t)$ and is spatially Gaussian in shape. The measurement of the spatio-temporal evolution of the temperature field induced by this excitation, with the help of an infrared thermography camera, makes it possible to go up, with the help of a mathematical post-treatment, to the value of the thermal effusivity of the material.

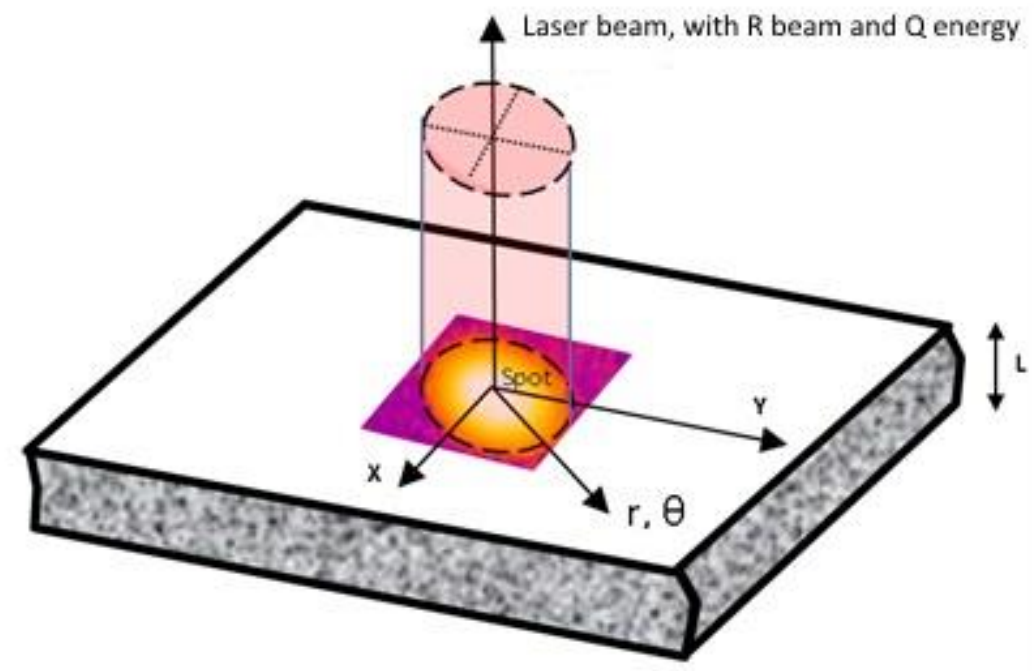

Fig. 1. The operating method implemented for the study

Let us examine in detail this mathematical post-processing on which this measurement technique is based. Either a plate of thickness $L$, semi infinite in $r$. Or a very brief thermal disturbance (Dirac delta function $\delta(t)$ ), and spatially Gaussian shape. This excitation is applied at the initial time $t=0$ in the center of the plate in order to avoid edge effects. Let $R$ be the characteristic radius of this excitation task (measured at $\mathrm{Q}_{\max } / \mathrm{e}^{2}$ ). Let be $\lambda, \rho, c$ and $a$, respectively, the thermal conductivity, the density, the calorific capacity and the thermal diffusivity of the material constituting the studied plate. The sample is initially in thermal equilibrium with its environment. Finally, in this model we will neglect the convecto-radiative exchanges between the sample studied and the environment. The mathematical translation of these assumptions leads to the following differential system:

$$
\begin{gathered}
\Delta T(r, z, t)=\frac{1}{a} \frac{\partial T(r, z, t)}{\partial t} \\
\text { En } \mathrm{z}=0:-\lambda \frac{\partial T(r, 0, t)}{\partial t}=\frac{2 Q}{\pi R^{2}} \operatorname{Exp}\left(-\frac{2 r^{2}}{R^{2}}\right) \delta(t)
\end{gathered}
$$




$$
\begin{gathered}
\text { In } \mathrm{z}=\mathrm{L}:-\lambda \frac{\partial T(r, L, t)}{\partial t}=0 \\
\text { At } \mathrm{t}=0: \quad \mathrm{T}=\mathrm{T}_{\text {ext }}
\end{gathered}
$$

The resolution of this differential system implements two integral transformations on one hand a Hankel transformation of order 0 on the $r$ axis (4):

$$
H_{0}\left[\frac{1}{r} \frac{\partial}{\partial r}\left(r \frac{\partial T(r, z, t)}{\partial r}\right)\right]=-\sigma^{2} \int_{0}^{\infty} r . J_{0} .(\sigma \cdot r) T(r, z, t) . d r
$$

and on the other hand, a Fourier transformation with respect to time (5) :

$$
F\left(\frac{\partial T(r, z, t)}{\partial t}\right)=\frac{i \cdot \omega}{\sqrt{2 \cdot \pi}} \int_{-\infty}^{+\infty} T(r, z, t) \cdot \operatorname{Exp}(-i \cdot \omega \cdot t) \cdot d t
$$

It leads to the expression of the spatio-temporal evolution of the temperature on the front panel of the studied sample given by formula (6):

$$
T(r, 0, t)=\frac{2 Q}{b \sqrt{\pi^{3} t}} \cdot \frac{1}{R^{2}+8 a t} \cdot \operatorname{Exp}\left(\frac{2 r^{2}}{R^{2}+8 a t}\right)
$$

E Now considering the experimental conditions conventionally encountered in our study: i.e. an excitation radius of $R=1 \mathrm{~cm}$, a thermal diffusivity a equal to $8.15 .10^{-7} \mathrm{~m}^{2} / \mathrm{s}$ and an analysis time $t=0.2 \mathrm{~s}$, the term 8at becomes negligible in the term $\mathrm{R}^{2}$ and the formula (6) then becomes :

$$
T(r, 0, t) \approx \frac{2 Q}{b \sqrt{\pi^{3} t}} \cdot \frac{1}{R^{2}} \cdot \exp \left(-\frac{2 r^{2}}{R^{2}}\right)
$$

If the measurement is now made at the center of the thermal spot, i.e. in $r=0$, then this expression becomes :

$$
T(r, 0, t) \approx \frac{2 Q}{b \sqrt{\pi^{3} t}} \cdot \frac{1}{R^{2}}
$$

By now posing $Q^{\prime}=\frac{2 Q}{\pi R^{2}}$ it is possible to rewrite the previous expression in the form :

$$
T(t)=\frac{Q^{\prime}}{b \sqrt{\pi t}}
$$

One then finds an expression rate close to that obtained during a thermal effusivity estimation by an extended flash excitation. To arrive at a local estimate of the thermal effusivity parameter, we propose, as in this case, to implement a comparison method. Thus, by depositing the same energy on the sample to be analyzed and on a known effusivity reference sample, it is possible to estimate the desired effusivity using the following formula :

$$
b_{\text {unk }}=\frac{T_{r e f}(0, t)}{T_{u n k}(0, t)} \cdot b_{r e f}
$$




\section{The samples studied and the experimental system implemented during the study}

For our study, we had at our disposal 6 calibrated samples. These are two PVC samples (A sample and reference sample), one bakelite sample (B sample) and three nylon samples ( $C$ to $E$ sample). Their geometric dimensions are as follows: a length of $40 \mathrm{~mm}$ a width of $40 \mathrm{~mm}$. The reference sample is $10 \mathrm{~mm}$ thick and the other five are $3 \mathrm{~mm}$ thick. They were all coated with a thin layer of black paint to homogenize their radiative properties (Figure 2).

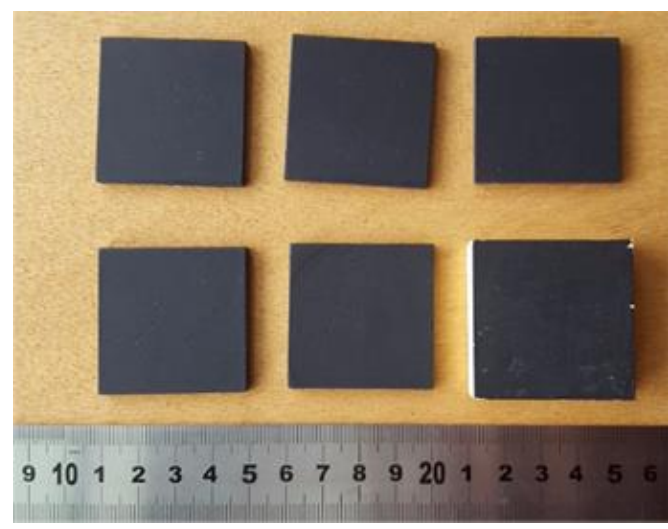

Fig. 2. Studied samples

Their thermal effusivity was measured using the DICO device - described in the following publications $[4,5]$ - of the CERTES laboratory at the University of Paris Sud. The values provided by this laboratory are grouped in Table 1.

Table 1. The effusivity values measured using the DICO device of the CERTES laboratory.

\begin{tabular}{|l|l|l|l|l|l|l|}
\hline & $\begin{array}{l}\text { PVC } \\
\text { reference }\end{array}$ & Sample A & Sample B & Sample C & Sample D & Sample E \\
\hline $\begin{array}{l}\text { b reference } \\
\left(\mathrm{J} . \mathrm{m}^{-2} \cdot \mathrm{K}^{-1} \cdot \mathrm{s}^{-1 / 2}\right)\end{array}$ & $\mathbf{4 0 8}$ & $\mathbf{3 4 5}$ & $\mathbf{4 7 1}$ & $\mathbf{3 5 8}$ & $\mathbf{5 5 5}$ & $\mathbf{6 1 1}$ \\
\hline
\end{tabular}

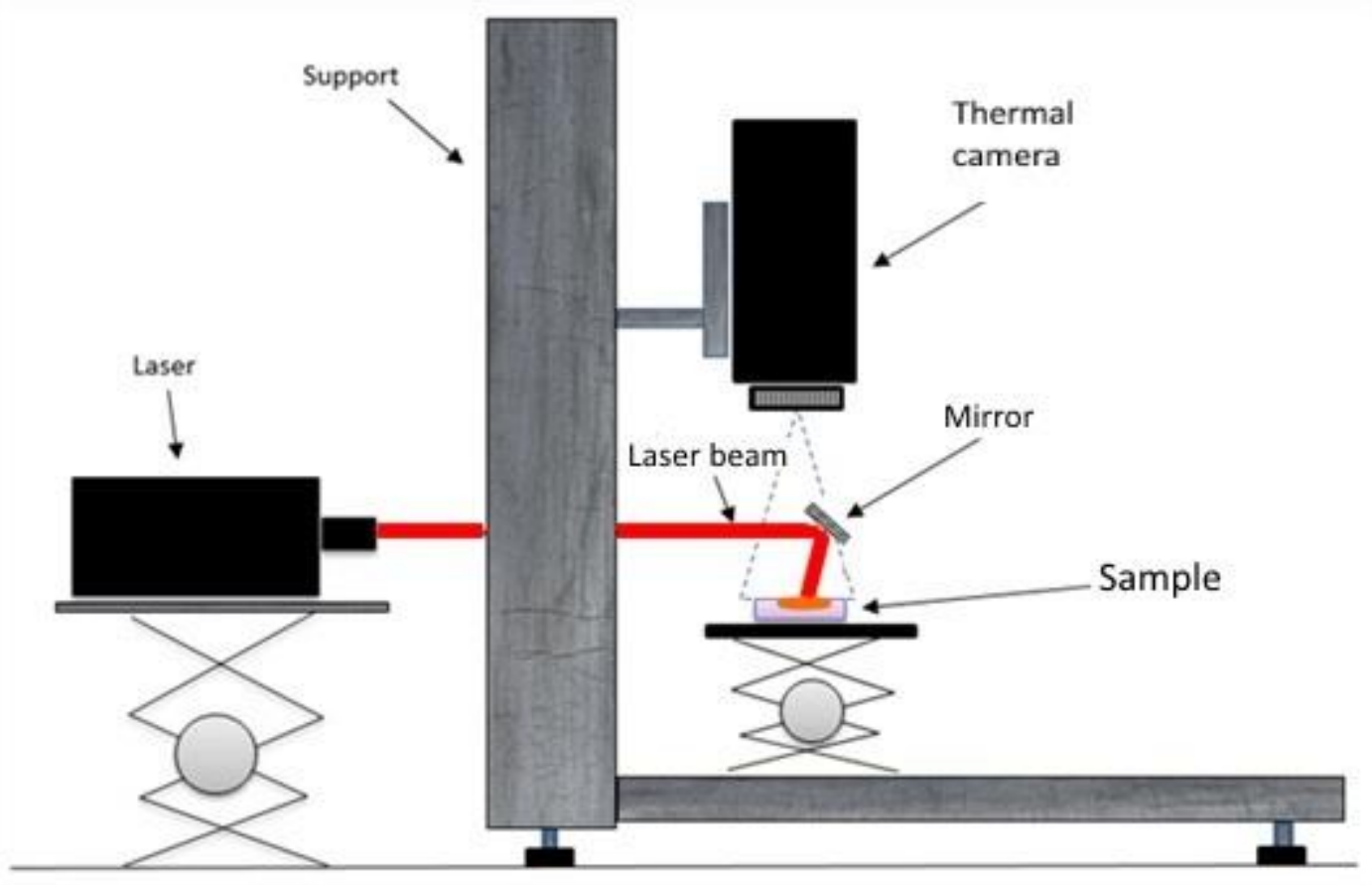

Fig 3. Schematic of the experimental set-up implemented 
The experimental device used is first of all a mechanical stand made of microcontrolled profiles. The base of this stand is a plate full of duralumin $40 \mathrm{~cm}$ wide and $50 \mathrm{~cm}$ long. Its thickness is equal to $2 \mathrm{~cm}$. These dimensions have been chosen to ensure good stability of the experimental disc device. This plate is placed on height-adjustable feet to allow fine adjustment of the flatness of the experiment. At one end of this plate is fixed a $95 \mathrm{~mm}$ side and $85 \mathrm{~cm}$ high microcontrol Xprofile. Two jumpers are positioned on this profile. The first allows the fixation of the infrared thermotherapy camera. The second, equipped with a fine movement microcontrol screw allows a precise positioning of the infrared thermography camera.

The experimental device implemented is then composed of an excitation optics. It is an AMS type laser diode. Its maximum power is $3.5 \mathrm{~W}$. Its emission wavelength is $0.810 \mu \mathrm{m}$. It is controlled by control and synchronization electronics with the infrared thermography camera. The laser is positioned on a microcontrol device allowing its fine positioning. An aluminium deflecting mirror, placed at approximately 45 degrees, allows the exciation of the sample studied.

Finally the experimental device implemented is composed of an infrared thermography camera. This is a FLIR SC655 bolometer camera with a macro thermographic lens. These choices are justified by the important time constants of the samples studied and by the centimetric dimensions of the laser excitation (figure 3 ).

\section{Experimental results obtained}

The six samples were studied using this experimental device. The experimental conditions selected are the following: an excitation duration of $20 \mathrm{~ms}$, an acquisition frequency of $100 \mathrm{~Hz}$ and finally an excitation radius of $1 \mathrm{~cm}$. From the thermographic films obtained in this framework, we then drew the temporal evolution of the temperature at the centre of the thermal trace. An example of the results obtained is shown in Figure 4. It shows well a $-1 / 2$ slope behavior during the first 200 milliseconds of the analysis, which justifies the simplications taken into account previously.

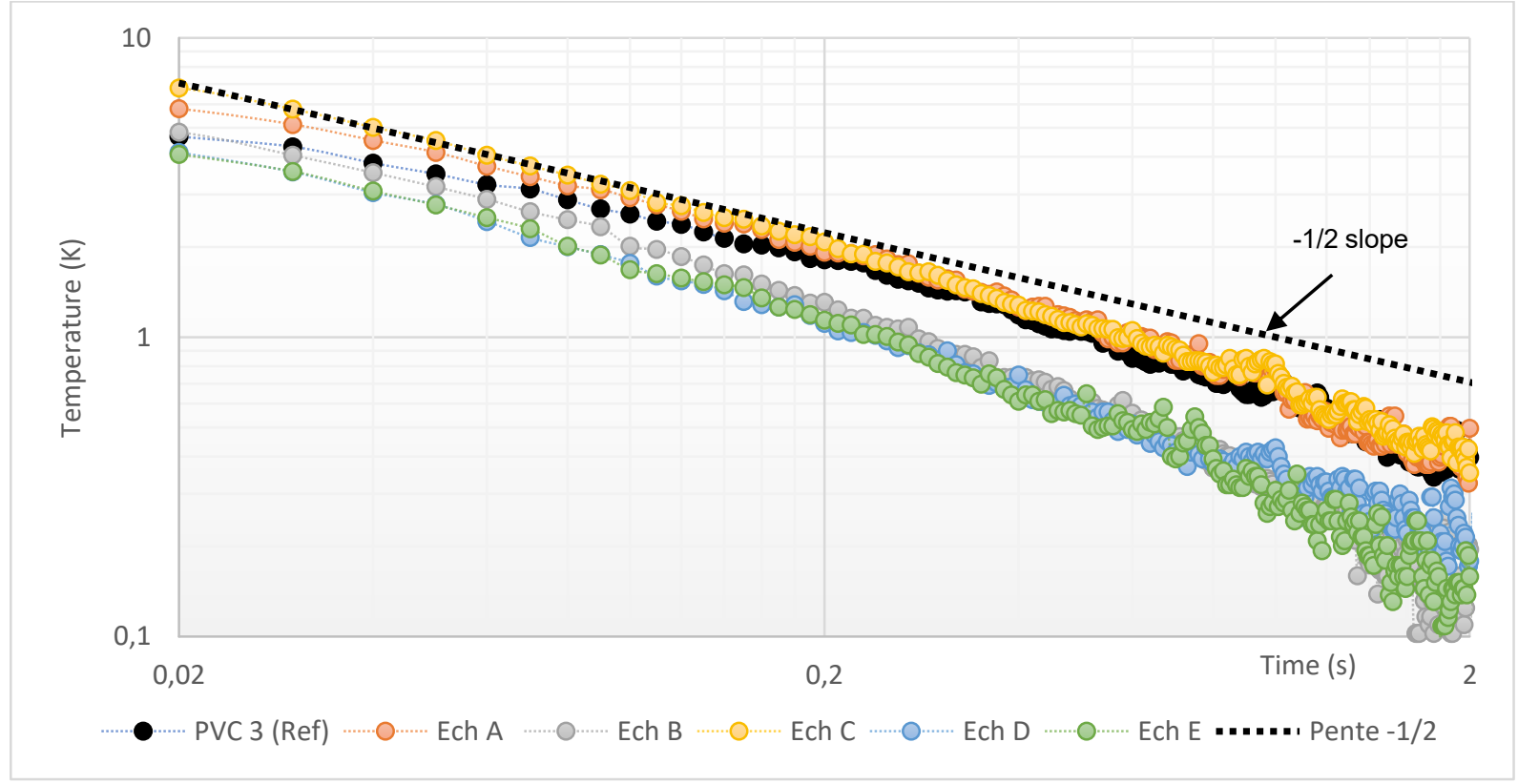

Fig 4. Temperature responses of samples (Log-Log representation)

From these curves and using the comparison method, we estimated the effusivities of the $A, B, C, D$ and $E$ samples; the ref sample of course serving as a reference. The results obtained are presented in Table 2 . They show that the estimated thermal effusivity values are close to the reference values and therefore the feasibility of the photothermal method.

Table 2. The experimental results obtained

\begin{tabular}{|l|l|l|l|l|l|}
\hline & Sample A & Sample B & Sample C & Sample D & Sample E \\
\hline $\begin{array}{l}\text { b estimated } \\
\left(\mathrm{J} \cdot \mathrm{m}^{-2} \cdot \mathrm{K}^{-1} \cdot \mathrm{s}^{-1 / 2}\right)\end{array}$ & 343 & 466 & 334 & 543 & 563 \\
\hline $\begin{array}{l}\text { DICO reference } \\
\left(\mathrm{J} \cdot \mathrm{m}^{-2} \cdot \mathrm{K}^{-1} \cdot \mathrm{s}^{-1 / 2}\right)\end{array}$ & 345 & 471 & 358 & 555 & 611 \\
\hline Relative gap \% & $-0,5 \%$ & $-1,0 \%$ & $-6,8 \%$ & $-2,1 \%$ & $-7,9 \%$ \\
\hline
\end{tabular}


The measurements obtained correlate well with the expected baseline measurements. The relative differences do not exceed $12 \%$ which is very encouraging.

\section{Conclusion}

Photothermal methods are an important tool for the detection and characterisation of structural defects in the conservation of works of art. Within the framework of hygroscopic salt pollution detection, it is the study of the local evolution of thermophysical properties that we propose to implement. However, the thickness of the wall paintings and the need for in-situ measurements led us to introduce a longitudinal thermal effusivity estimate. In this work, we first presented our investigation approach. It is based on the study of the lateral diffusion of a heat spot generated by a laser pulse. We then developed a model of this approach and showed that in the case of works of art, thermally slow, that the method of comparisons used classically in extended flash front panel method is applicable in our case study. In a third stage, we presented the experimental set-up implemented for the study. Finally, with the help of 5 test samples, previously characterized by the laboratory, we have shown the feasibility of the proposed method. This study should now be generalized to a wider range of samples. The reproducibility and robustness of the photothermal method should still be studied. Finally, its possibilities for detecting hygroscopic salt pollution in works of art of the cultural heritage should be studied. Studies in this direction are under way.

\section{REFERENCES}

[1] Bodnar, J. L., Candoré, J. C., Nicolas, J. L., Szatanik, G., Detalle, V., \& Vallet, J. M. (2012). Stimulated infrared thermography applied to help restoring mural paintings. NDT \& E International, 49, 40-46.

[2] Bodnar, J. L., Mouhoubi, K., Di Pallo, L., Detalle, V., Vallet, J. M., \& Duvaut, T. (2013). Contribution to the improvement of heritage mural painting non-destructive testing by stimulated infrared thermography. The European Physical Journal-Applied Physics, 64(1).

[3] Cernuschi, F., Russo, A., Lorenzoni, L., \& Figari, A. (2001). In-plane thermal diffusivity evaluation by infrared thermography. Review of scientific instruments, 72(10), 3988-3995.

[4] Krupa, I., Boudenne, A., \& Ibos, L. (2007). Thermophysical properties of polyethylene filled with metal coated polyamide particles. European Polymer Journal, 43(6), 2443-2452.

[5] Sobolciak, P., Karkri, M., Al-Maadeed, M. A., \& Krupa, I. (2016). Thermal characterization of phase change materials based on linear low-density polyethylene, paraffin wax and expanded graphite. Renewable Energy, 88, 372-382. 